\title{
FAULT LOCATION STRATEGIES ON POWER CABLES USING OFFLINE METHODS INCLUDE TIME DOMAIN REFLECTOMETRY ( TDR)
}

\author{
Ali Hassan Ibrahim Mansour \\ Elect. Eng. Dept., Faculty of Eng. Al-Azhar University, Qena, Egypt
}

\begin{abstract}
The purpose of this study is to investigate the feasibility of offline fault location and state of health technologies for airport series circuits used to provide power to light fixtures on the runway. In current series circuits, only some ground faults can be detected and no automated fault location or state of health features are available. Fault location is currently performed with an isolation tester and a"divide and conquer"-methodology which is very time consuming and personnel intensive.
\end{abstract}

\section{INTRODUCTION \\ 1.1 Background}

Since air traffic is expected to double within the coming 15 years [1], safety and efficiency of airports are subjected to new challenges. The airfield lighting system (AFL) consists of LED-lights which are connected in long loops which can be several kilometers long. One of the challenges with maintaining the cable circuits is to detect and locate different intermittent faults. Common causes of faults are water and moisture intrusion due to faulty splices or connectors.

ADB-Safegate is one of the industry leaders in automated airside systems today, and they offer a variety of solutions for airports. Their portfolio includes automated docking systems and smart airfield lighting systems. Safegate has sold equipment to airports worldwide, [1]

The paper Investigate offline fault location methods and state of health. Different computer tools for simulation and calculations were used. To estimate attenuation in transmission lines and transformer frequency response LTSpice [2] was used. LTSpice was also used when simulating faults on the series circuit. For post processing, the data obtained from LTSpice simulations was imported into Matlab where all the calculation were made

\subsection{Problem Statement}

In AFL systems today, the light fixtures are connected in series while being decoupled from the series circuit using isolation transformers. The series circuits are designed to operate for several years and have proven to be very reliable. Although, occasionally the AFL suffers from electrical faults. The constant current regulator (CCR) is able to measure resistance to ground and successfully detect some electrical faults, but lacks the possibility to pinpoint and locate faults. Therefore, the lack of a fault locating system requires that airport traffic is temporarily suspended and that a maintenance team is dispatched out to the runway to pinpoint the fault location. This is done by using offline methods measuring one segment at a time which can be very time consuming when a fault occurs far out on the series circuit. A fault location system would greatly minimize traffic suspension time and thus reduce maintenance costs for electrical faults on the cable system. 


\subsection{Goal}

The purpose of this paper is to try and meet the increasing demands on automation and operational safety of the airport. The paper work involves development and evaluation of methods that can detect and localize electrical faults on the series circuits, and possibly also determine the state of health of the system.

The method should be able to operate on series circuits of all lengths and loads. In order to ensure compatibility with older series circuits it should not require series circuit communication.

The goals of the paper work is to fulfill the following criteria:

- Perform a feasibility-study of the potential of existing fault location and state of health technologies and identification of factors that influence the possibility for future implementation in an AFL- system.

- Perform a technical evaluation and comparison of existing fault location and state of health methodologies for future implementation in an AFL-system.

\subsection{Time Domain Reflectometry}

The Megger TDR is able to produce $20 \mathrm{~V}$ with pulse lengths between $2 \mu \mathrm{s}$ and $2 \mathrm{~ns}$, which provides an effective range of a few meters up to $20 \mathrm{~km}$. It is possible to adjust velocity factor and characteristic impedance to optimize the TDR equipment for specific cable systems.

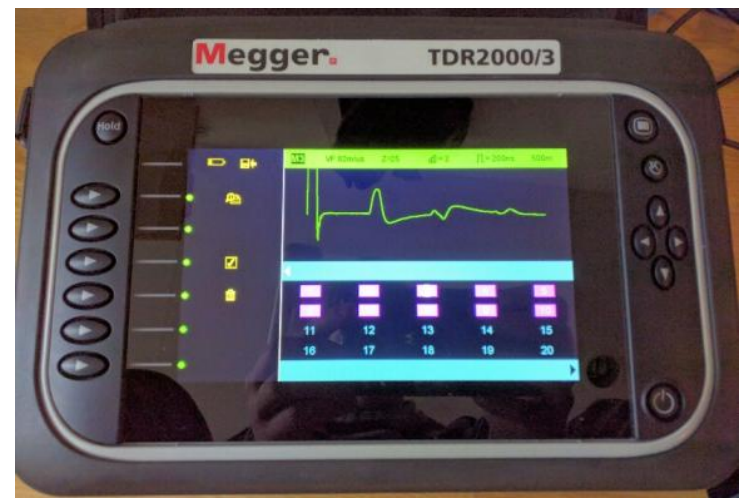

Figure 1: Megger TDR.

Since the TDR does not requires high voltage equipment it has been tested both in a simulated environment as well as with a real commercial TDR (Megger TDR2000/3). The lab cable length was validated in order to adjust the velocity factor of the TDR equipment. The velocity factor is specific for different cable types, and has to be validated prior to performing fault location with TDR. Three different setups has been evaluated. Measurements on a transmission line without, with one and with two transformers.

\section{Airfield Lighting System}

The airfield lighting system is used to light up runways and taxiways on airports around the world, it consists of a constant current regulator that provides power to the primary cable. Along the primary cable, isolation transformers are placed in series, where the secondary winding of each transformer is connected to an individual light fixture. [1]

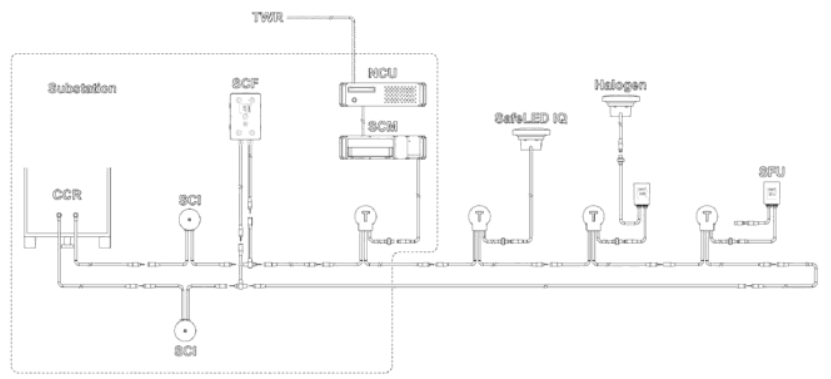

Figure 2: AFL-series circuit setup

\subsection{Constant Current Regulator}

The constant current regulator (CCR) is an AC-AC converter consisting of a phase controlling thyristorbridge and a high voltage transformer that can transform the voltage from grid-level up to $5 \mathrm{kV}(50-60 \mathrm{~Hz})$. The CCR keeps a constant output current of $6.6 \mathrm{~A}$ by regulating the voltage level in order to follow load 
changes on the series circuit. The CCR is currently able to measure earth-impedance and the minimum limit is $50 \mathrm{M} \Omega$, but the CCR is not able to locate the fault. These earth-impedance measurements can be routed to a central SCADA system which displays the data to airport personnel. [1]

\subsection{Primary Series Circuit}

The primary series circuit consists of a single pole coaxial cable. The primary cable can be shielded or unshielded depending on local requirements at each individual airport. The primary series circuit connects the CCR to each individual light fixture by using isolation transformers to decouple the lights from the primary series circuit, this allows other lights in the series circuit to continue operating if one or more light suffers from breakdown.

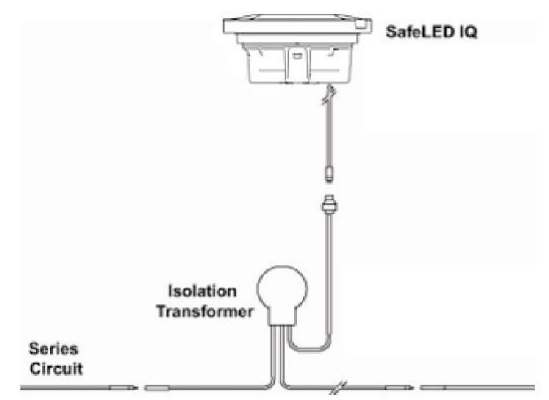

Figure 3: Light/Transformer-connection.

[4]

\subsection{Isolation Transformers}

Current transformers are generally used in metering and over-current relay protection applications. But, in AFL-systems, they are used to overcome the"Christmas tree light"-problem. Since all light fixtures are connected in series, the entire series circuit will break down if 1 fixture fails. Utilizing current transformers to decouple the light fixture from the series circuit maintains a current path on the series circuit that will ensure operation of the remaining light fixtures. When a light fails, the secondary circuit becomes open and the transformer is saturated due to current still running through the primary winding.

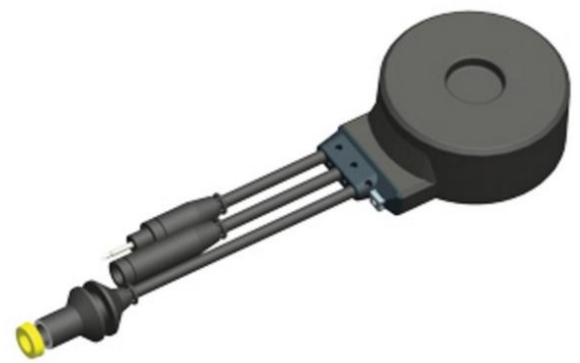

Figure 4: Isolation Transformer from EFLA. [3]

The EFLA transformers recommended by Safegate to their customers are rated at 6.6A rms and from $10 \mathrm{~W}$ to $500 \mathrm{~W}$. Transformers are often "over-sized" in relation to their load in order to lengthen the lifetime of the transformer. [2]

\subsection{Light Fixtures}

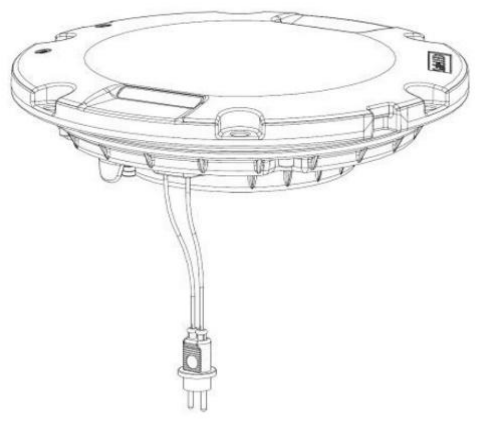

(a) Safe LED, Inset-Light [1]

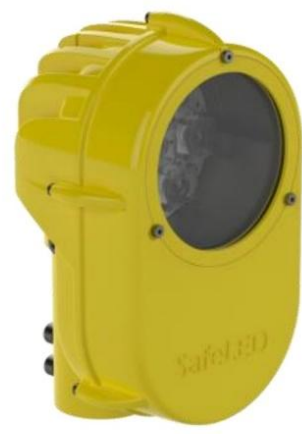

(b) Safe LED, Elevated Light 
There are a number of different light fixtures available from Safegate, which are designed for different applications on the airfield. They can be categorized in 2 different types, elevated light fixtures and inset light fixtures. Inset lights are designed to be implemented into the ground on the runway and to have minimum vertical intrusion up onto the runway, while the elevated lights are usually placed on the edge of the runways and taxiways where vertical intrusion is of less importance.The fixtures are available with both LED and halogen type lights, halogen lights are significantly less efficient and are continuously being phased out of airports. The power level of the lights depend on light intensity. Although, the maximum power rating for these lights is $58 \mathrm{~W}$ per light fixture for the LED type. [1]

\subsection{Airfield Smart Power}

Each light in the AFL-system can be controlled individually by utilizing the Series Circuit Modem (SCM). The SCM is mounted along the series circuit according to Figure 2. The SCM superimposes high frequency communication on to the $50 / 60 \mathrm{~Hz}$ power frequency. By doing so, the number of transmission lines are kept to a minimum. This technique is called power line communication (PLC).

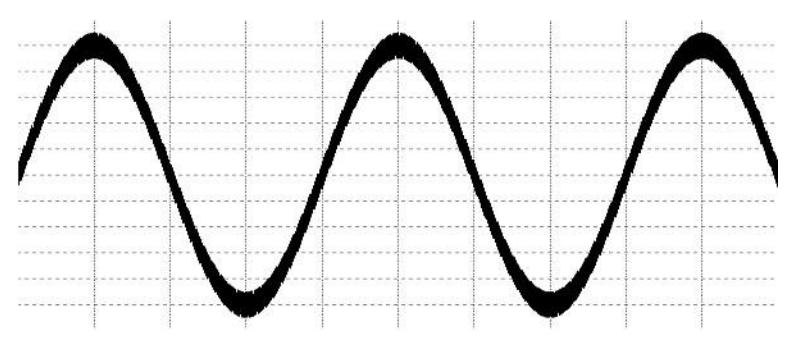

Figure 6: Super-imposed communication on carrier signal.

Since some of series circuits are intelligent and bi-directional communication is possible using the SCM, individual light control and monitoring is possible. The operator can then be notified of individual light faults, location and fault type for fast and easy maintenance. This system is called Airfield Smart Power (ASP). [1]

\subsection{Series Circuit Filtering}

In order to protect the constant current control loop of the CCR from external and communication noise, filtering is required. Therefore, the series circuit filter (SCF) and series circuit inductance (SCI) are implemented at the terminals of the series circuit.

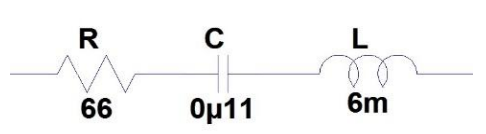

(a) Series Circuit Filter

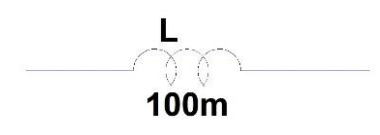

(b) Series Circuit Inductance

\section{Coaxial Cable Structure}

Figure 7: Series Circuit Filterin

The underground transmission line that have been subject to testing in the lab and simulations in LTspice is shown in fig. (1)

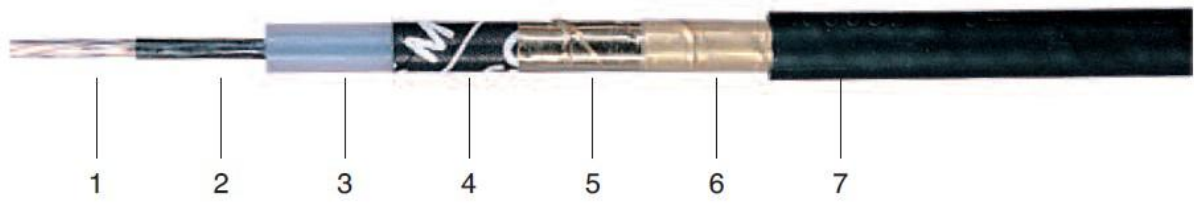

Figure 8: Coaxial Transmission Line from EUPEN. [3]

1. Stranded tinned copper conductor

2. Extruded semi-conducting compound

3. XLPE Insulation

4. Semi-conducting tape, helically applied

5. One lay of brass tape, helically applied, $30 \%$ overlapping

6. Separation Tape

7. PE outer sheath - black 
As can be seen above, the cable consists of a copper conductor, then followed by a semiconducting layer, XLPE insulation, yet another semiconducting layer and then finally the conductive shield, which is grounded. The outer sheath usually consists of a PVC plastic to provide chemical, UV and weather resistance. Since the magnitude of the electrical field is equal to the voltage difference between two points, the semi-conductive layers relieve voltage stress along the crossover points between insulation and conductor where the voltage stress is especially high. [4]

Table 1: Physical Parameters, EUPEN Cable. [3]

\begin{tabular}{|r|r|r|r|r|}
\hline Cross-Section & Insulation Thickness & Screen Tape & Sheath Thickness & Overall Diameter \\
\hline $1 \times 6 \mathrm{~mm}^{2}$ & $2.3 \mathrm{~mm}$ & $0.08 \times 22 \mathrm{~mm}$ & $1.6 \mathrm{~mm}$ & $11.5-13.0 \mathrm{~mm}$ \\
\hline
\end{tabular}

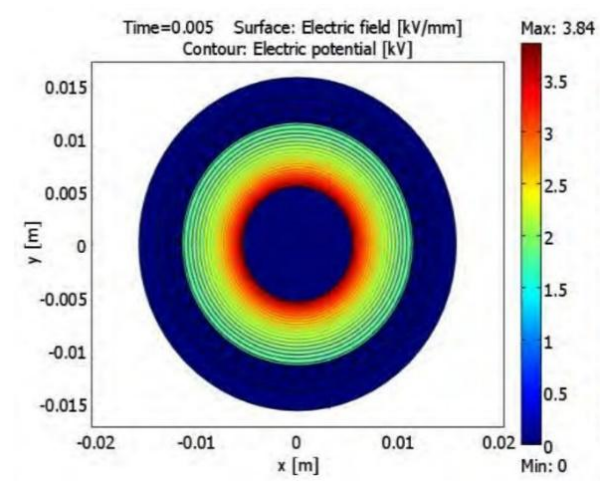

Figure 10: Equipotential electrical field distribution of a shielded coaxial cable. C[2012] IEEE [18]

\subsection{Electrical Faults}

In an AFL-system fault event, there are 3 consecutive states of operation.

1. Pre-Fault Steady State

2. Fault Transient State

3. Post-Fault Steady State

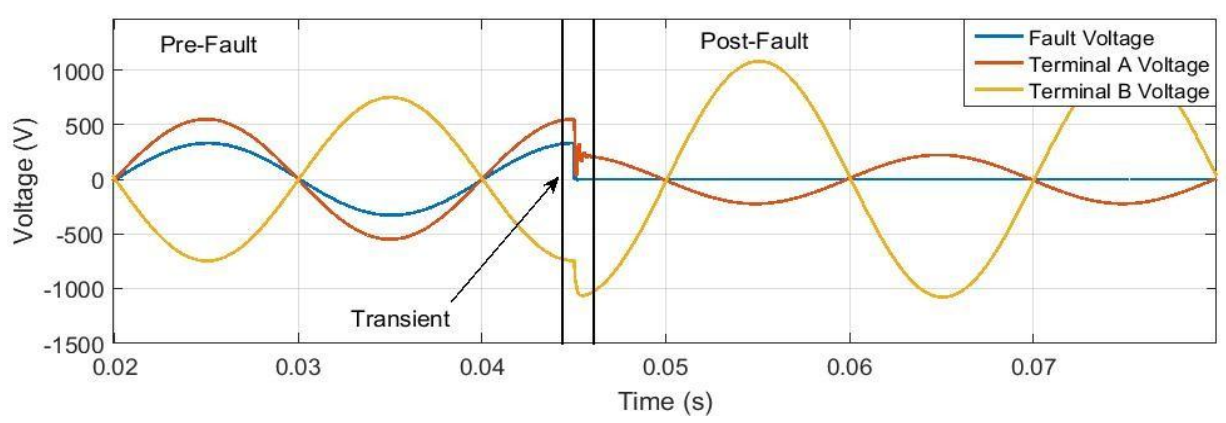

Figure 9: Illustration of fault states of a bolted ground fault, with a current source.

Before a fault, the system is in normal steady state operation. When a fault occurs and the system enter the transient fault state, a voltage impulse is generated at the fault location and steady state is disturbed, the energy stored in the distributed L and $\mathrm{C}$ parameters of the transmission line starts to oscillate. Eventually, the oscillations die out and we obtain the post fault steady state, where the system has adjusted to the parameters of the new faulted circuit. 


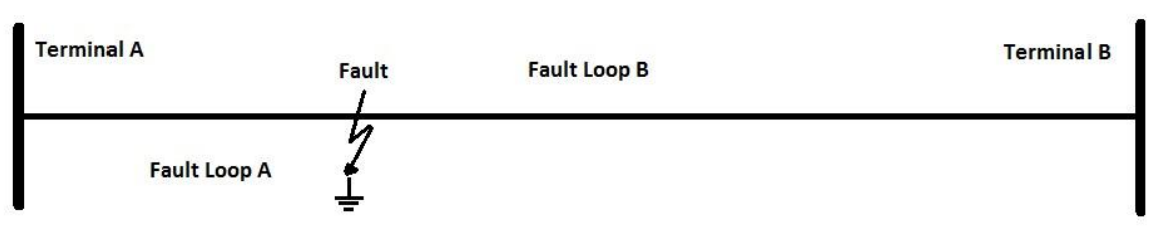

Figure 10: Schematic of post fault state.

The post fault steady state parameters will be decided by the location of the fault. When a fault occurs, two fault loops will be obtained. One loop from terminal A to fault, and another from terminal B to fault. As can be seen in fig $16, \mathrm{~V}_{\mathrm{a}}<<\mathrm{V}_{\mathrm{b}}$. Which means that the fault is significantly closer to terminal $\mathrm{A}$ than to terminal B.

A fault in an electrical transmission system can have a number of different causes, these can be completely external or depend on the electrical operation. Electrical faults can be described as a spark gap in parallel with a nonlinear resistance appearing in a cable segment. The fault resistance is nonlinear, and can be voltage dependent. But, for fault location techniques a basic linear fault model will suffice. [9] The fault can either be categorized as a series fault where the core conductor is subjected to a unwanted discontinuity, or a ground fault (shunt fault) where core conductor and the concentric shield establishes a low resistance path. In practice, an electrical fault can also be a combination of a shunt and series event. [6]

Generally for overhead lines, faults can disappear by themselves since the arc can self-extinguish, this is usually not the case for underground cables. When the insulation suffers a breakdown, a low resistance path is formed between conductor core and sheath (conductive shield). This produces a ground fault, which can either stay as a ground fault or develop into a series fault. A series fault is developed if the cable is completely burned apart. In order for a ground fault to be established in an underground system, insulation has to break down, either due to mechanical or electrical disturbances.

Insulation can suffer breakdowns for a number of reasons. When insulation is subjected to electrical stress, voids and impurities in the insulation material can give rise to a phenomenon known as electrical treeing which can start to propagate in the insulation until it causes a fault. If moisture or water is present, another phenomenon known as water treeing can start to degrade insulation quality, even under low electrical stress. Mechanical intrusion can also cause a fault, this can be due to improper handling of the cable during construction of the AFL-system or even due to rodents eating through the cable.

Given that the cable is energized at fault inception, a fault event will generate fault waves that will travel from the fault location towards the terminals of the transmission system. The fault wave is generated due to the instantaneous change of voltage and current at the moment of fault. [7]

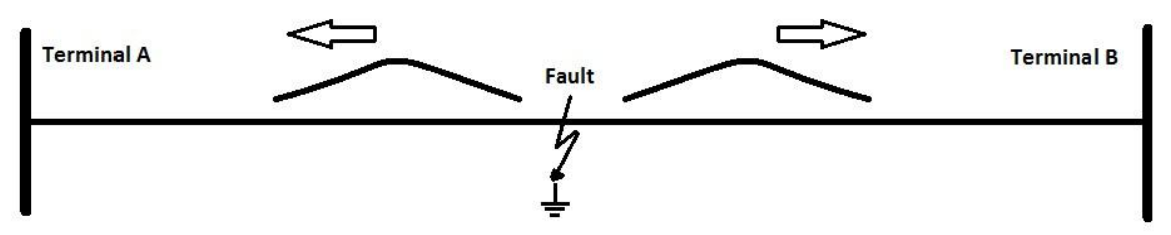

Figure 11: Illustration of fault waves.

\subsubsection{Ground Faults}

When insulation has degraded to the point of breakdown, there are 3 possible fault scenarios. Either a short circuit develops, this can be due to mechanical damage that has forced conductor and sheath into contact, or a carbon-metal bridge has formed between the two layers providing a fault resistance $<5 \Omega$ due to burnt cable insulation. The insulation can also evaporate and provide a gaseous low resistance path between conductor and sheath. A fault with resistance $<5 \Omega$ is referred to as a bolted fault. [10] In the case of an AFL-system where the source is a CCR, the source will eventually adjust to the post fault steady state and produce a 6.6A output current.

Critical ground faults which affect flight operations at airports are very unusual, these occur a few times a year. A fault of this magnitude would be in the same order as bolted faults. The majority of ground faults are rather small, usually in the order of a few $100 \mathrm{k} \Omega$. Smaller faults do not affect daily operations, but can potentially develop into a critical fault and therefore affect flight operations in the future. 


\subsubsection{Series Faults}

A series fault in a coaxial cable will occur when the core conductor is severed. In order for this to happen in a coaxial cable, a ground fault must first occur where core and sheath is in contact first. The transformation time from ground to series fault, can vary significantly. Causes for series faults can be mechanical damage where the sheath and/or conductor has been severed, electrical causes include ground faults which have been allowed to blow apart the entire coaxial cable. [6]In splices and connectors, a series fault can occur without first undergoing a ground fault event. Water intrusion and

faulty splice connections can cause the connector surfaces to oxidize. Aluminum and copper are two conductors whose oxidized surface has a higher resistance than the base metal. Therefore in faulty splices where the quality of the oxidization-inhibitors has been compromised, c o ntact-resistance can be significantly higher. [8]

In an AFL-system, a series fault is considered more dangerous than a ground fault. During a ground fault, voltage will be adjusted down in order for the CCR to output 6.6A. But, when a series fault occurs, the CCR will have to increase voltage in order to maintain rated current output. This will produce dangerous excess voltage on the series circuit.

\subsubsection{Intermittent Faults}

An intermittent electrical fault is a fault which is not permanent, but which occurs periodically or seemingly sporadic. This can be due to partial insulation breakdown at peak voltages, or splice glitching. A partially faulty splice could suffer from glitching due to water or moist intrusion or due to mechanical fatigue. The electrical manifestation of a intermittent fault can vary significantly depending on the type. If a splice is mechanically fatigued, the fault will not show unless it is mechanically stressed. In an AFLsystem for instance, this is possible if an aircraft is taking of or landing and thus producing substantial vibration in the area surrounding the runway.

\subsubsection{Fault Inception Angle}

The fault inception angle is the angle at which a fault occurs $\left(0<\phi_{\text {fault }}<2 \pi\right)$, and is of high importance when analyzing and discussing the potential of different fault location techniques. In OHL-systems a fault is entirely independent of the fault inception angle, since faults are caused by exterior events such as lightning strikes or falling trees. In underground transmission lines, internal cable faults are caused by electrical stress, meaning that a coaxial cable fault is statistically more likely to occur close to peak voltages. According to Flytkjaer Jensen and data from Energinet.dk, all faults caused by internal cable failure occurs with a maximum deviation of $25^{\circ}$ from peak voltage. [7] Although, since AFL-systems are not completely buried, rather inserted into cable ducts between manholes, the system is still vulnerable to external causes of failure. For instance, rodents and water or moisture intrusion. The size of the fault waves generated by the fault, is entirely dependent of the fault inception angle. If a fault occurs when the momentary voltage at the fault location is $0 \mathrm{~V}$, no fault waves will be generated.

\section{Fault Location Strategies}

Fault location systems have been in use in standard electrical transmission systems for decades. FL- systems play an important role in regard to power continuity, reliability and power quality. Since an electrical fault in general cause mechanical damage, fast fault localization enables efficient equipment repairs and sometimes even makes it possible to utilize pre-emptive maintenance. The importance and interest of FL-systems have recently grown due to technical and economic advances in low-cost DAQ and computation systems. [5]

In the present day, there are a multitude of different fault location technologies in use, all of which are developed for the standard commercial grid. These technologies can be categorized into offline and online methods. Online methods consists of two types of methods, impedance-based and traveling wave based methods. An online method works autonomously to approximate a fault using DAQsystems and signal processing. The signal processing involved, can be more or less advanced. With the recent rise of artificial neural networks (ANN) and fuzzy logic, fault location has been identified as a possible application. Offline methods include time domain reflectometry (TDR), high voltage pulse surging and visual inspection. These methods are considered to be very accurate, but extremely time consuming and requires extensive personnel training. [7]

The relative error obtained from a fault location method can be written as follows:

$$
\text { error }(\%)=\frac{\mathrm{d}-\mathrm{d}_{\text {exact }}}{1} * 100 \%
$$


where, $d$ is the fault location obtained from the FL-algorithm, $d_{\text {exact }}$ represents the actual fault location and 1 is the total electrical length of the transmission line.

The general roots of fault location errors which applies for all fault location methods are incorrect fault classification and insufficient or inaccurate data assumptions. Usually, the total transmission line length is only known with some error, or transmission line parameters are effected by outer factors such as weather and soil resistivity. [6]

The fault location method cannot affect or disturb ongoing operation on the series circuit. In order for it to be backward compatible with previous Safegate solutions it cann ot require ASP communication between fixtures since older series circuits does not provide this feature. Therefore the online methods have been limited to utilizing the terminals of the CCR

\subsection{Impedance Based Methods}

In electrical grids today, there are one- and two-ended impedance methods in use today. One ended methods are used in situations when both terminals are not in the same geographical position, and communication is needed between the two to utilize two-ended methods.

Impedance based FL is structured so that one can sample voltage and current phasors at one or both terminals. The obtained data is then inserted into algorithms that produce an approximated fault location based on circuit equations. Since these algorithms utilize circuit parameters, a substantial amount of data has to be known about the transmission line including line parameters and distance approximations.

An impedance based fault locator requires a phasor measurement unit (PMU) in order for terminal currents and voltages to be measured at power frequency and calculates an estimated fault location. The algorithms requires phase-to-ground voltages and phase currents at the terminals. [9]

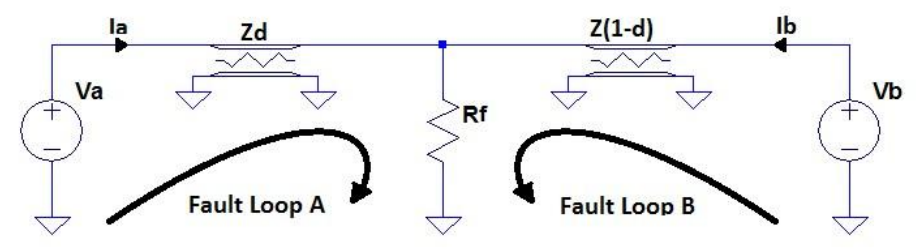

Figure 13: Fault loop schematic of a ground fault.

As mentioned in section 3.4, when a ground fault occurs in a transmission line, two fault loops are obtained, one from each terminal. The fault loop seen from terminal A can be described with the following KVL-equation:

$$
0=\mathrm{V}_{\mathrm{a}}-\mathrm{dZ} \mathbf{I}_{\mathrm{a}}-\mathbf{R}_{\mathbf{f}} \mathbf{I}_{\mathbf{f}}
$$

Where $\mathrm{Z}$ is the distributed line impedance p.u, $\mathrm{d}$ is the distance p.u and $\mathbf{I}_{\mathbf{f}}$ is the fault current. If one measures the impedance at terminal $\mathrm{A}$ in a fault event using current and voltage phasors, a value of the apparent impedance from source to fault is obtained.

$$
Z_{f a}=\frac{V_{a}}{I_{a}}=Z d+R_{f} \frac{I_{f}}{I_{a}}
$$

The reactance method measures the impedance from source to fault, , utilizes the fact that the ratio of reactance to fault and the total reactance of the line is proportional to the distance to fault.

$$
d=\frac{\operatorname{Im}\left(V_{a} / I_{a}\right)}{\operatorname{Im}(Z)}
$$

By only utilizing the reactive component of impedance, the method is able to compensate for fault resistance. If If a is complex, a considerable reactance error will be introduced, since the reactance effect 
As can be seen in the equation above, the fault loop impedance is a definitive measure of the fault loop length. However, the fault impedance is unknown, so this can not come to any practical use unless the fault impedance is either very small or equal to zero. [5]

For the remainder of this section, two impedance based fault location algorithms will be presented:

- Reactance method (one ended)

- Synchronized \& un-synchronized phasor method (two ended)

- Voltage magnitude profile for non-homogeneous systems (two-ended)

affects the apparent line reactance to fault.

The synchronized/un-synchronized phasor method is a two ended method, which exploits the fact that data can be used from both terminals of the transmission system. By utilizing KVL from terminals A and B to fault, the following statement can be obtained.

$$
\left\{\begin{array}{l}
0=V_{f}+Z d I_{a}-V_{a} \\
0=V_{f}+Z(1-d) I_{b}-V_{b}
\end{array}\right.
$$

In the equation above, where all terms are phasors except the distance to fault $\mathrm{d}$ in p.u from terminal

A, which is a scalar. By subtracting one equation with the other, the fault voltage can be eliminated. if we solve for $d$ we will get:

$$
\mathrm{d}=\frac{\mathrm{V}_{\mathrm{a}}-\mathrm{V}_{\mathrm{b}}+\mathrm{Z} \mathbf{I}_{\mathrm{b}}}{\mathrm{Z}\left(\mathbf{I}_{\mathrm{a}}+\mathrm{I}_{\mathrm{b}}\right)}
$$

According to IEEE, this FL algorithm provides a way to find the fault location by using post-fault phasor data. Although, if the measurements are unsynchronized, this requires the system to have reached steady state, i.e that the fault resistance is constant. If the fault impedance is time varying, this will lead to different magnitudes and phase angles at the terminals. Therefore, synchronized phasor measurements will provide all four phasors (voltage and current at both terminals) at a specific time stamp, which will correspond to one specific fault impedance. Otherwise, errors are introduced and accuracy is compromised. [9]

The methods described above are mainly applicable to homogeneous transmission line systems. The algorithms will have to be altered if they are to be used on an AFL-system.

In metropolitan areas, transmission lines are usually a combination of OHL- and undergroundlines, a so called hybrid transmission line. In order to cope with non-homogeneous lines, Gong et al. developed a two ended impedance based FL methodology which utilizes current and voltage measurements at the terminals along with characteristics of the TL to establish a voltage profile from each terminal and thus find the fault location at the intersect of those two curves. [10] 


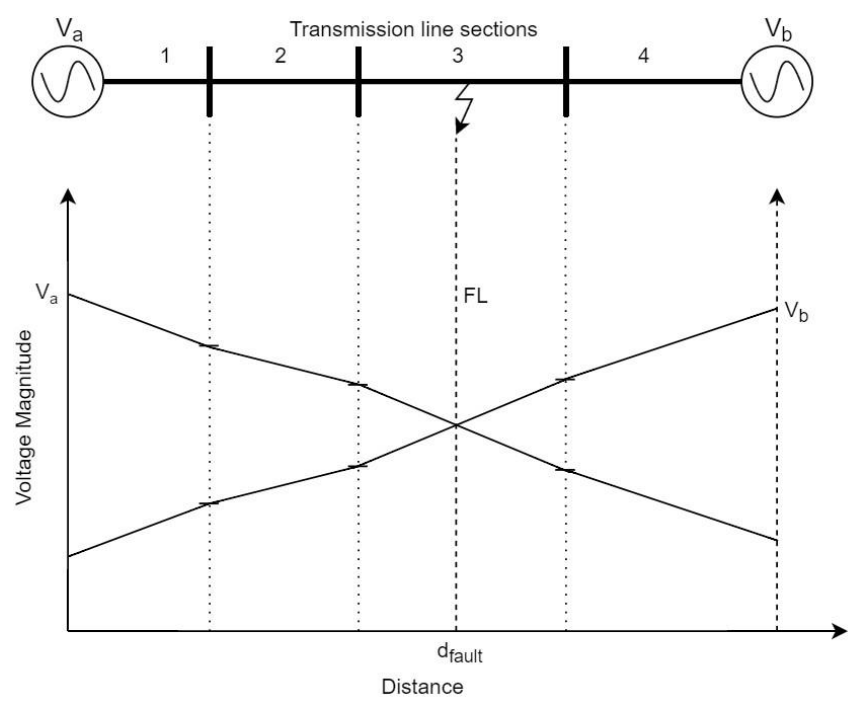

\subsection{Time Domain Reflectometry}

Figure 14: Voltage profile of a fault hybrid line.

Time domain reflectometry (TDR) is a fault location method, which transmits low-voltage pulses with short time duration into the cable and analyzes the reflections coming back to the reflectometer. It is essential that the cable is shielded, since the method requires a conductor and a sheath of equal electrical length. The pulse is often a short square wave with a low voltage of about $20 \mathrm{~V}$. As the pulse travels through the cable it will be reflected due to deviations from the characteristic impedance of the cable. Visible impedance discontinuities can consist of cable ends, splices, transformers and cable faults. An open end will only reflect the wave but a shorted end or ground fault will reflect and inverted pulse (figure 15).

When a pulse is transmitted into the cable the distance between cable opening and the discontinuity or irregularity can be determined from a reflection-time display. The display shows the transmitted and all the reflected pulses at the time when they arrive back to the TDR. An example can be seen in figure 22 .

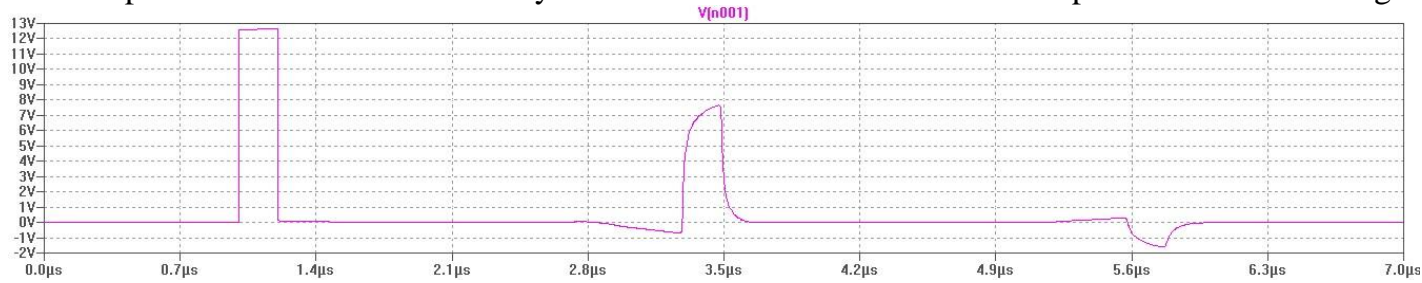

Figure 15: Simulation of how the pulse is transmitted into a cable with an open end. The pulse is transmitted at time $1 \mu$ s and reflected from the open end at $3.2 \mu \mathrm{s}$.

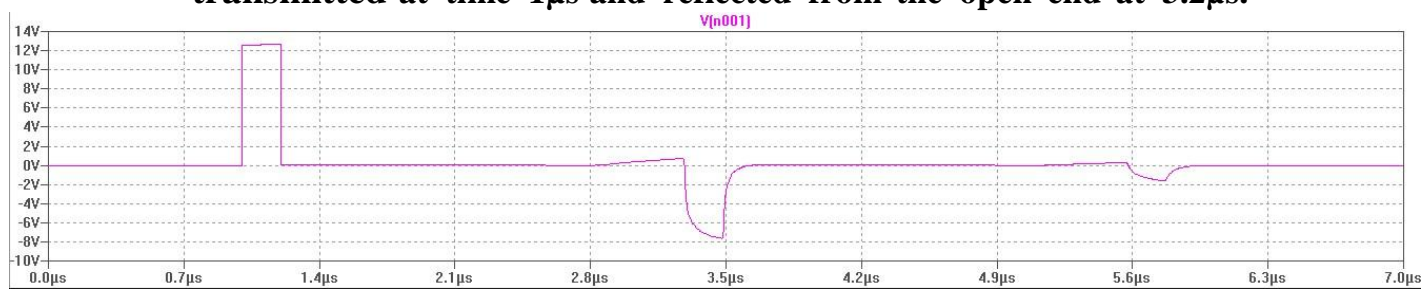

Figure 16: Simulation of how the pulse is transmitted into a cable with a shorted end. The pulse is transmitted at time $1 \mu \mathrm{s}$ and reflected and inverted from the shorted end at $3.2 \mu \mathrm{s}$

With only a TDR, it is not possible to locate ground faults with resistance values greater than ten times the characteristic impedance of the cable or intermittent cable faults [9]. Therefore, high resistance ground faults may become problematic to locate. Furthermore, due to high frequency attenuation, any reflections obtained from long distances will be severely attenuated.

Main factors influencing fault location accuracy are:

- The length of the cable

- Incorrect approximation of velocity factor

- Excessive reflections from unwanted impedance discontinuities 


\subsection{High Voltage Pulse Surging}

High voltage pulse surge (HVPS), also called capacitive discharge or thumping, is a method as the name implies transmitting a high-voltage pulse into the system. The pulse itself will introduce a large potential over the fault on the cable in order to generate an arc in between the gap of the fault (either conductorconductor or to conductor-earth). That arc will generate energy into its surroundings in form of sound waves and electro-magnetic waves that can be heard or detected by electronic sensors or even the human ear.

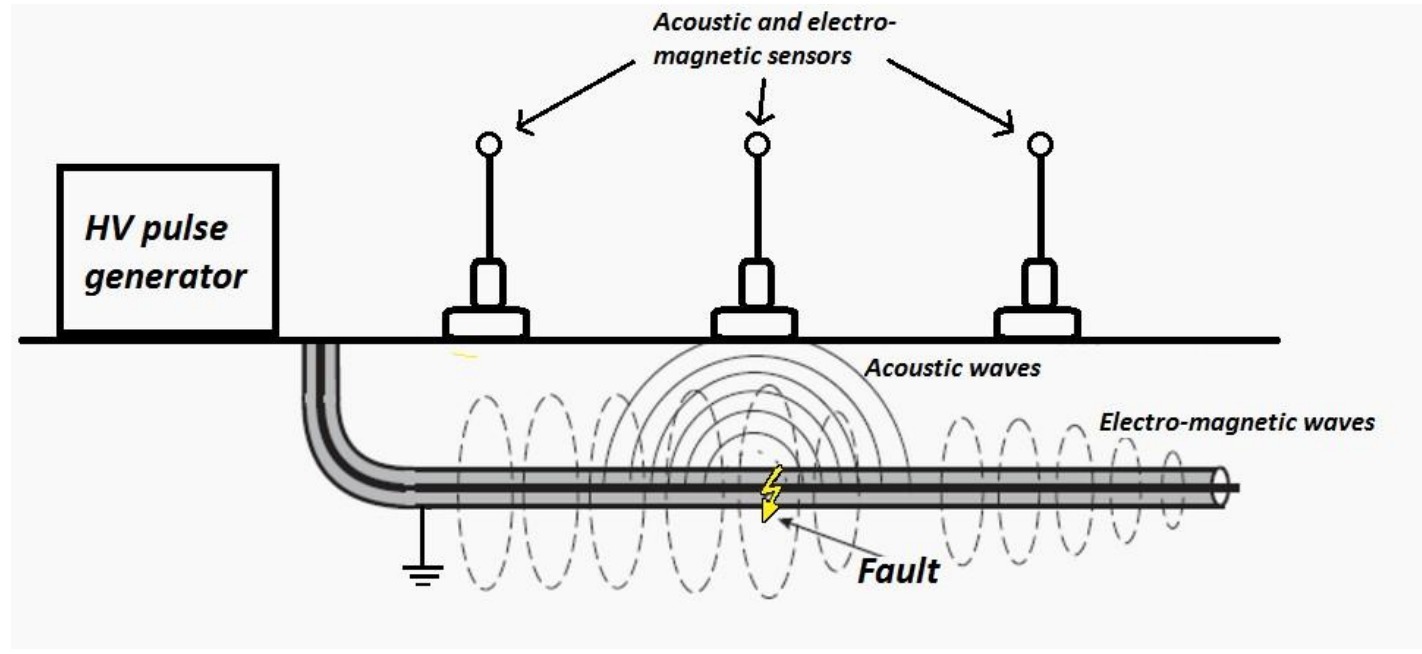

Figure 17: When sending in the HV pulse the fault will generate both electro-magnetic and acoustic waves that can be detected with sensors.

\subsection{Traveling Wave Methods}

In order to overcome the limitations of traditional fault location technologies, traveling wave based methods have been adopted for use in the electrical grid in recent years. The method exploits the correlation between backward-and forward-propagating waves in a medium. When a fault event occurs in a transmission line, if the inception angle is different from 0 , a fault wave will be initiated in both directions of the transmission line. Ideally, this fault wave is a perfect square wave containing a multitude of frequency components. Due to the high propagation velocity and frequency content of the fault wave, high sampling frequency is of utmost importance. The recent improvements in DAQ and communication systems have allowed for extremely high sampling rates and processing of large amounts of data, which have made traveling wave methods more relevant in recent years. Traveling wave methods are considered to be more accurate and less dependent on system parameters than traditional FL-methods, but implementation is more costly.

In a homogeneous medium, the fault wave travels at some fraction of light speed, in a coaxial cable the propagation velocity is determined by the distributed line parameters (section 3.2.6). As the wave travels through the medium, it is subjected to attenuation and thus, the wave energy decreases. When the fault wave encounters an impedance discontinuity, it is reflected and refracted according to the reflection coefficient of the discontinuity. [5]

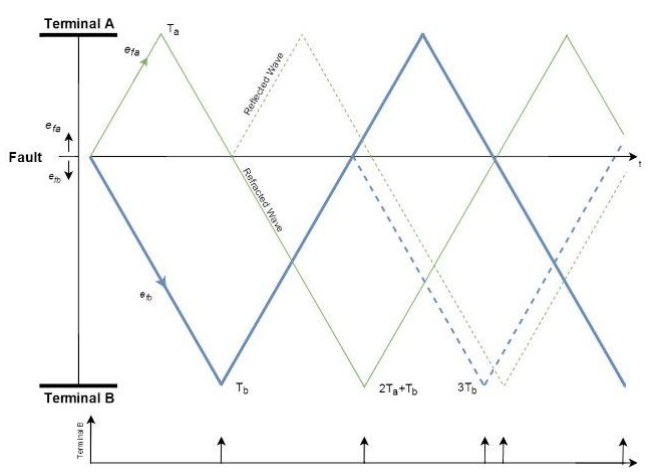

Figure 18: Lattice diagram of traveling wave in a homogeneous medium generated from a ground fault. 
As can be seen in the lattice diagram in fig 25 , the fault waves travel towards the terminals, reflects and refracts at the discontinuities. If the total line length (l) is known, the fault location $d_{\text {fault }}$ can be calculated in $[\mathrm{m}]$ from terminal A for a one ended method.

$$
d_{\text {fault }}=\frac{l}{2}+\frac{c V_{f} \Delta t_{a}}{2}
$$

Where $\mathrm{c}$ is the light speed in vacuum, $\mathrm{V}_{\mathbf{f}}$ is the velocity factor of the medium. $\Delta \mathrm{t}_{\mathrm{a}}$ is the time difference between the incidental wave at terminal A and the first reflection. For a two ended method:

$$
d_{\text {fault }}=\frac{l}{2}+C V_{f}\left(t_{b}-t_{a}\right)
$$

$\mathbf{t}_{\mathrm{a}}$ and $\mathrm{t}_{\mathrm{b}}$ are the respective arrival times of the traveling waves at terminal $\mathrm{A}$ and $\mathrm{B}$.

Traveling wave-technologies are generally categorized according to their mode of operation or design: Types A, B, C, D and E. Type A, utilizes a one ended implementation where the incidental and fault wave is observed as well as the wave reflected from the opposite terminal. Although, ty pe A can be problematic if there are multiple discontinuities on the line, since it can be difficult to distinguish the different wave fronts from each other. Type D on the other hand, utilizes a two ended implementation, where the incidental waves at both terminals are recorded. This eliminates the problem of distinguishing multiple reflections from each other since only the "first" transient is required to determine the fault location. In standard grid systems, the two ended method requires accurate time tagging at both terminals (done via GPS) and a communication system to bring the data to a centralized computing device. Type $\mathrm{C}$ is an active one ended method, which sends a pulse into the transmission line and records the reflection response of the system. This method is also known as TDR, which is treated as a separate method in this study (section 3.5.2). Type $\mathrm{E}$ is a one ended method that records the transients produced when the transmission line is re-energized when the circuit breakers at the terminals are closed. Traveling wave equipment that is used today, runs simultaneously in type A and D. If two ended methods are used, it is only a matter of detecting the fault waves. For systems with a large amount of attenuation, the fault waves can be attenuated to the point where measurement equipment cann ot detect the waves. [9]

The accuracy of a traveling wave FL-method is influenced by a number of factors. Firstly, $\mathrm{s}$ a mpling rate. The sampling frequency of the measurement equipment is directly correlated to the error of the transient detection. If a device samples at $1 \mathrm{MHz}$, the maximum detection error for a 2 terminal TW FL will occur when the incidental wave at each terminal is detected exactly 1 sample apart. Therefore, if we assume that $\mathrm{V}_{\mathbf{f}}=0.62$, the maximum error will be $86 \mathrm{~m}$ [11]. Furthermore, propagation velocity for very high frequency signals are slightly frequency dependent. According to C.K Flytkjaer Jensen, a wave evaluated at $1 \mathrm{M} \mathrm{H} \mathrm{z}$, induces an error of $6 \mathrm{~m}$ for a $25 \mathrm{~km}$ cable and $12 \mathrm{~m}$ for a $50 \mathrm{~km}$ transmission line. Therefore, a constant propagation velocity can be assumed for the transmission lines in this study. [7]

GPS-based traveling wave FL systems suffer in accuracy due to the uncertainty of the GPS time tagging. Measurement transducers can also give errors if they are saturated or for any other reason fail to reproduce the transient waveforms. One problem that can result in incorrect fault alarms can be incorrect wave detection, this is due to the fact that there can be other sources of waves which the FL system can not distinguish from an actual fault. In order to counter this problem one can utilize advanced signal processing to learn the transient signature of that specific system. [9]

\subsection{Wavelet Transform}

The Wavelet transform (WLT), is a mathematical transform which allows for time-frequency analysis. The uncertainty principle states that a high time resolution will result in a low frequency resolution and vice versa. Unlike the Fourier transform where a window of constant size is used, the WLT utilizes a variable window sizes to overcome the uncertainty principle. [11] The WLT has recently gained attention in power engineering due to the possible uses within transient signal and analysis, voltage distortion analysis, power quality analysis and monitoring, and power system protection. Although, the WLT is applicable in any scenario where non-stationary signals and transients are of interest. [5]

The wavelet transform on the signal $s(t)$ is defined as follows:

$$
C(a, b)=\int_{-\infty}^{\infty} s(t) \frac{1}{\sqrt{a}} \psi *\left(\frac{t-b}{a}\right) d t
$$


Where $\mathrm{a}$ and $\mathrm{b}$ is the scale and time shifting factors. $\psi^{*}$ is the time shifted and scaled mother wavelet $\psi(\mathrm{t})$. A discretization of the WLT can be described as:

$$
C\left(a, i T_{s}\right)=T_{s} \frac{1}{\sqrt{|a|}} \sum_{n=0}^{N-1}\left[\psi *\left(\frac{n T_{s}-i T_{s}}{a}\right) s\left(n T_{s}\right)\right]
$$

Where $\mathrm{N}$ is the total number of samples, $\mathrm{i}$ is an integer describing the number samples the daughter wavelet $\psi^{*}$ is shifted in time. $\mathrm{T}_{\mathrm{S}}$ is the sampling time.

Whereas the Fourier transform breaks a signal up into sine waves at different frequencies, the WLT divides the signal $s(t)$ into a multitude of shifted and scaled versions of the original wavelet (mother wavelet).

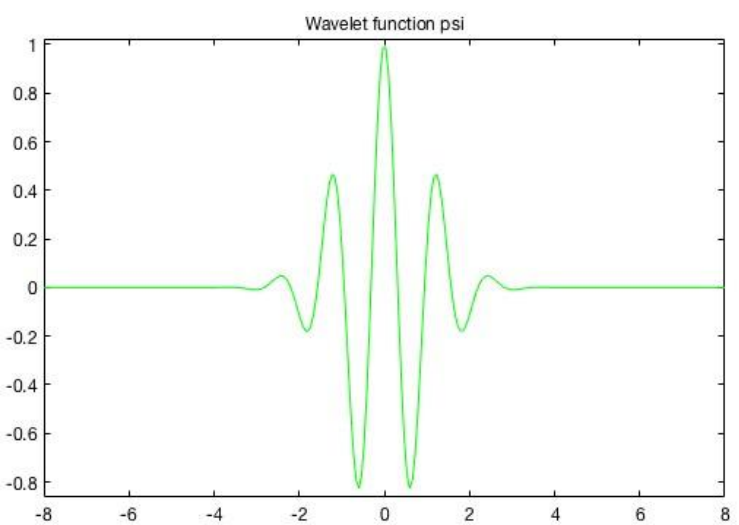

(a) Morlet Wavelet

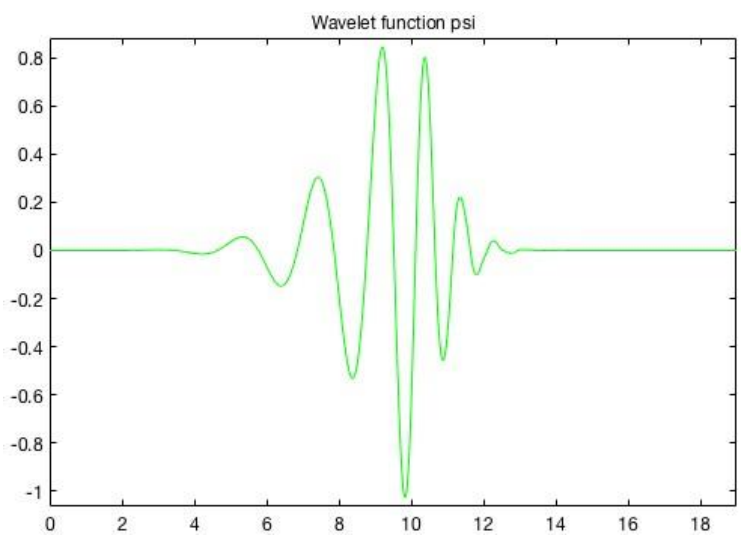

(c) Dubieties Wavelet

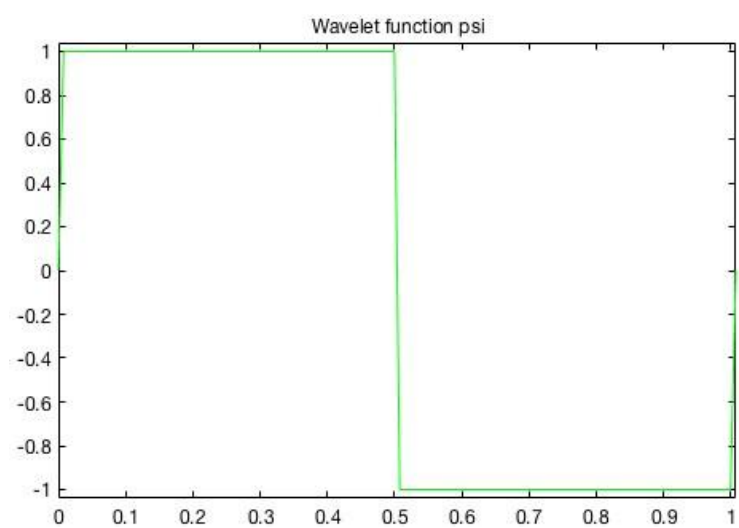

(b) Haar Wavelet

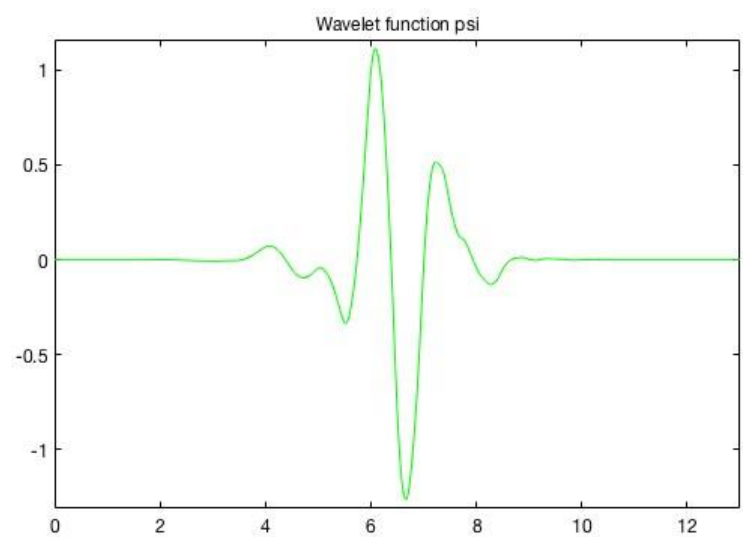

(d) Symlet Wavelet

Figure 18: Four of the more popular wavelets used in transient analysis.

The center frequency of a wavelet, is stated as "the frequency at which the modulus of the Fourier components of the mother wavelet is maximized" [7]. This means that the center frequency captures the dominant oscillation for that specific wavelet. By adjusting the scale (time window) of the wavelet, different frequency bands can be captured. This allows one to decompose the original signal into several signal components at different frequency bands.

Generally speaking, low scales containing high frequencies are considered to be more accurate but can be affected by noise. This means that transients which do not originate from the fault can muddle the result. High scales containing low frequencies which is less accurate, can be sensitive to power frequency, therefore fault transient characteristics may be lost. Dispersion effects may also influence the arrival time of fault waves. [11]

Considering the discussion above, Zhang [11] recommends utilizing high scales to detect the fault and it's approximate location, then applying a low scale high frequency decomposition to extract fault information and more precise fault location data. 

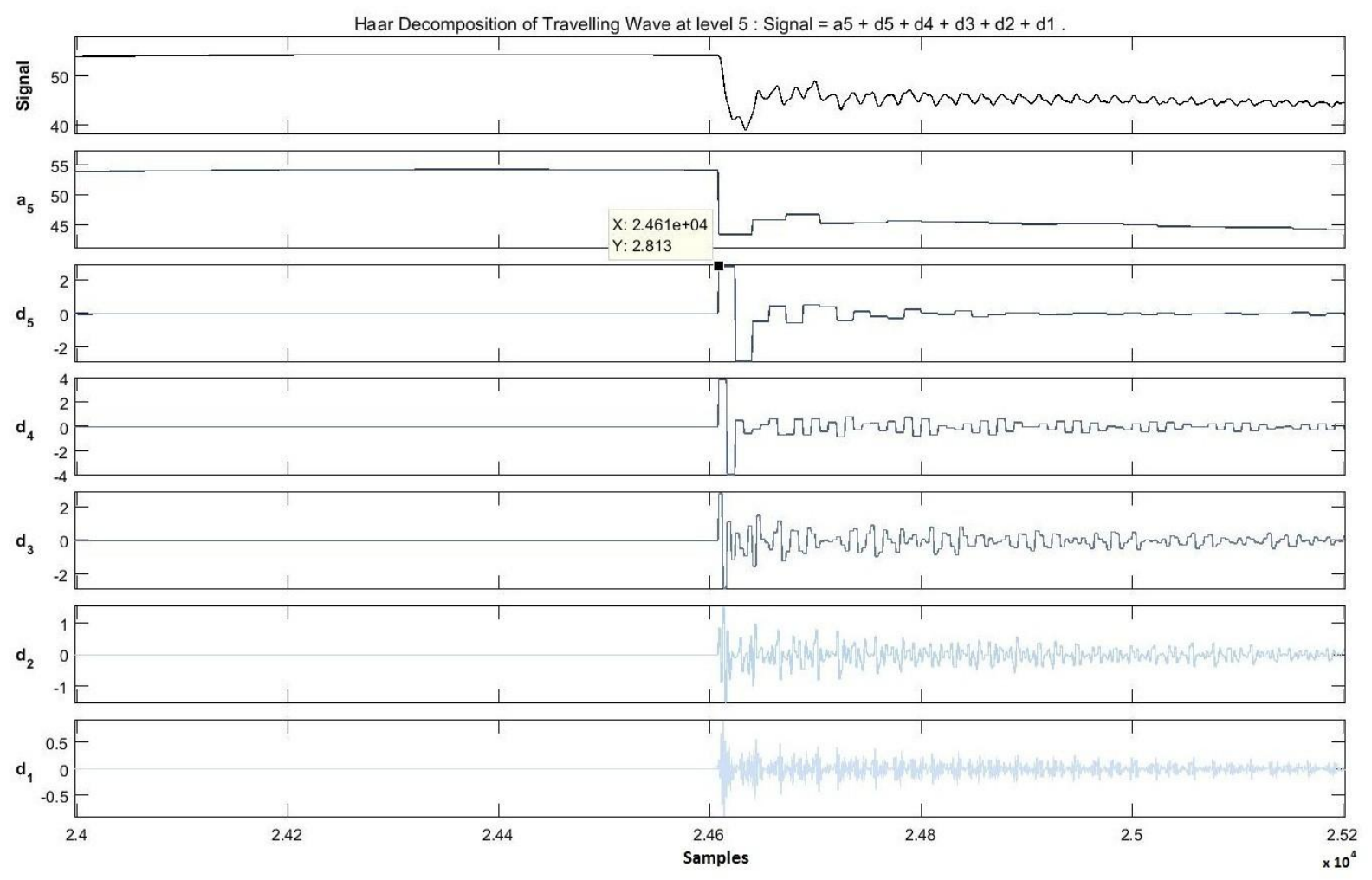

Figure 19: Voltage fault wave, haar Wavelet Decomposition at different scales. $F_{S}=1 M H z$

In fig 19, a voltage fault wave is displayed as "signal". The signal is then decomposed into coefficients of each scale. As can be seen, each decomposition coefficient captures a frequency band. Coefficient $a_{5}$ captures the overall trend while $d_{1}$ and $d_{2}$ encompass the very high frequency behavior. The Haar wavelet, whose psi function is a step function from 1 to -1 (fig 26b) can be seen in the figure above. This is especially visible in coefficient $a_{5}$ and $d_{5}$ where each progression in the coefficients is discrete.The result in fig 19 shows that it is possible to obtain high time resolution of fault waves, given that the sampling frequency is sufficient. Although, in a noisy environment where several random transient occur, additional signal processing is required to distinguish between signals which do not originate from faults and thus isolate the fault waves. The "signature" of the system has to be learned.

\subsection{Artificial Neural Networks \& Fuzzy Logic}

Artificial neural networks (ANN) and fuzzy logic (Flog) are examples of soft computing, where precision and certainty are not the goals. Research within soft computing is an effort to mimic intelligence and allow for decision making and pattern recognition which would not be possible using hard computing. The qualities that have been of interest regarding the mimicry of intelligence is mainly the ability to make rational decisions, adapting to missing data and accumulating experience over a longer horizon of time. Conventionally, one can use hysteresis and threshold values to monitor a process and make that it operates "within limits". In more complex systems and scenarios, fault indicators can become ambiguous and thus obstruct fault detection using conventional techniques. Fault location on power networks has been identified as a possible application of AI where accuracy and efficiency of FL processes can be significantly improved.

Unlike Boolean logic, fuzzy logic provides a framework for determining relations between events and objects based upon ambiguous, imprecise or noisy information. The main idea with Flog is that nothing is" false" or "true". No well-defined boundaries exist for the application, and can therefore partly be false and true to some degree. Its application in FL algorithms is therefore of interest to the research community. In a fault event, there is an amount of information which cann ot be known in all scenarios, such as fault resistances and measurements cann ot always reproduce all possible characteristics of such an event. Furthermore, observation possibilities are limited due to the difference in geographical position and the number of observable parameters are restricted to terminal voltage and currents. [5] 


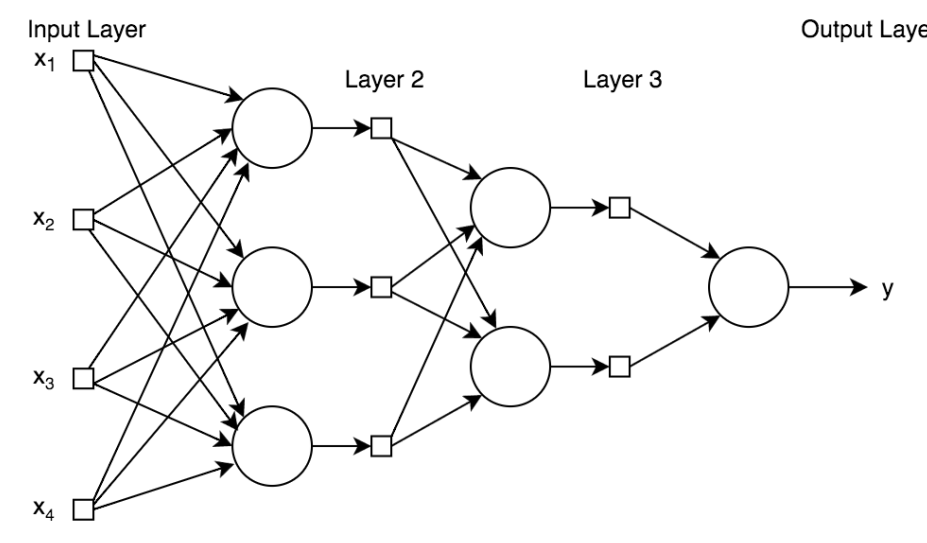

Figure 20: Multi-layer feed forward ANN architecture

ANNs are a structure of layers comprised of "neurons" which are interconnected. Each neuron performs an operation on the data it is fed from the input or previous neuron and then feeds it forward. ANNs mimic intelligence in the manner that it is trained by using previous scenarios to achieve a desired output, and therefore relies on previous experience.

Engadget reported in June of 2015 that AI researchers had used the video game Super Mario to train an ANN. In this case, the desired output was to maximize a "fitness level" that increased as the video game character progressed to the right, and the inputs were the pixels of the video game. As the "learning iterations" progressed, the AI found the right combinations of inputs to achieve the desired outcome, it was not aware of the video game in any way [12]. The same method of thinking can be applied to fault location algorithms. By designating a desired result, and showing an ANN data of fault events and allowing the ANN to accumulate experience from previous examples it is possible to utilize this method in real applications. [5]

Although, training an ANN for use in FL algorithms requires large amounts of reliable data. This data is not always available, and if data from simulations is used, there is a possibility for insufficient model assumptions which will impact algorithm performance.

\section{Conclusions}

The goal of this study was to perform a technical and operative feasibility study of fault location and SoH methods on airfield series circuits. The basis of the study was formed from a literature study of conventional fault location and SoH technologies, these methods were then applied and evaluated for a series circuit system. Due to design constraints such as backward compatibility and operation without PLC, the online methods were limited to utilizing CCR terminals.

AFL-systems differ from conventional grid systems. Firstly, a CCR will provide a different source behaviour than a source in a conventional grid. Furthermore, a series circuit consists of transformers as distributed loads along the circuit. Therefore, the effect of these system differences had to be evaluated for the methods of interest. Finally, the use of manholes transformer connections and splices allow for more frequent water and moisture intrusion than in a conventional grid, which makes the cable system more vulnerable than if the system would be completely buried.

\section{REFERENCES}

[1] Safegate Group .The future airport. Available: http://www. safegate.com/home/ The-future-airport, 2016-

[2] Linear Technology .LTspice. Available: http://www. linear. com/designtools/ Software/\#LTspice, 2016.

[3] Kabelwerk Eupen AG. Eupen Datasheet: FAA-L-824 - Type C - Brass tape screened $5 \mathrm{kV}$ [Data Sheet]. Available: http://www. eupen. com/weimages/airfield/primary\_cables/ faa-1-824\_type \_brass \_tape \_screened. pdf 2016-.

[4] K. Stein rich. IEEE Explore - Digital library. Influence of semiconducting la y e r s on the attenuation behavior of single-core power cables. Volume: 152, 2005.

[5] Saha, M.M., Iz y k ow ski, J., Rosolowski, E. (2010). Fault location o n power networks. London: Springer, 2010.

[6] IEEE Explore - Digital library (2007). IEEE Guide for Fault-locating Techniques on Shielded Power Cable Systems. New York: The Institute of Electrical and Electronics Engineers, 2007.

[7] Flytkjær Jensen, C. (2014). Online Location of Faults on AC Cables. Switzerland: Springer, 2014. 
[8] Frank, R., Morton, C. (2005). IEEE Explore - Digital library Comparative corrosion and current burst testing of copper and aluminum electrical power connectors, 2005.

[9] IEEE Explore - Digital library (2005). IEEE Guide for determining fault location on ac transmission and distribution lines. New York: The Institute of Electrical and Electronics Engineers, 2005.

[10] Gong, Y., Mynam, M., Guzman, A., Benmouyal, G., Shulim, B. (2012). Automated fa u lt location system for nonhomogeneous transmission networks. Schwitzerland: Schweitzer Engineering Labora - tories, Inc, 2012.

[11] Simon anderjon,dennis honbnen locating faults on energized airfield lightning power cables master thesis, june.2016

[12] Honkanen, Dennis Lu And Anderjon, Simon Locating Faults On Energized Airfield Lightning Power Cables (2016)In CODEN:LUTEDX/TELE EIE $0920 \quad 20161 \quad$ Industrial Elect rical Engineering And Automation $\checkmark$ Abbreviations and Indices

$\begin{array}{ll}\text { AC } & \text { Alternating Current } \\ \text { AFL } & \text { Airfield Lighting } \\ \text { AI } & \text { Artifical Intelligence } \\ \text { ANN } & \text { Artificial Neural Network } \\ \text { ASP } & \text { Airfield Smart Power } \\ \text { CCR } & \text { Constant Current Regulator } \\ \text { CT } & \text { Current Transformer } \\ \text { DAQ } & \text { Data Acquisition } \\ \text { SoH } & \text { State of Health } \\ \text { FL } & \text { Fault Location } \\ \text { FLog } & \text { Fuzzy Logic } \\ \text { HPC } & \text { High Power Converter } \\ \text { KCL } & \text { Kirchoff's Current Law } \\ \text { KVL } & \text { Kirchoff's Voltage Law } \\ \text { LED } & \text { Light Emitting Diode } \\ \text { OHL } & \text { Overhead Lines } \\ \text { PD } & \text { Partial Discharge } \\ \text { PLC } & \text { Power Line Communication } \\ \text { PMU } & \text { Phasor Measurement } \\ \text { Unit P.U } & \quad \text { Per Unit } \\ \text { PVC } & \text { Polyvinyl Chloride } \\ \text { SCADA } & \text { Supervisory Control and Data Acquisition } \\ \text { SCF } & \text { Series Circuit Filter } \\ \text { SCI } & \text { Series Circuit Inductance } \\ \text { SCM } & \text { Series Circuit Modem } \\ \text { SoH } & \text { State of Health } \\ \text { TW } & \text { Traveling Wave } \\ \text { TDR } & \text { Time Domain Reflectometry } \\ \text { UV } & \text { Ultraviolet } \\ \text { VT } & \text { Voltage Transformer } \\ \text { WLT } & \text { Wavelet Transform } \\ \text { Polyeth } & \end{array}$

